GTINDIA2014-8225

\title{
DESIGNING SUPERCRITICAL CO2 POWER PLANTS USING AN INTEGRATED DESIGN SYSTEM
}

\author{
Abdul Nassar \\ Dr. Maksym Burlaka \\ SoftlnWay Inc. \\ 15 New England Executive Park \\ Burlington, MA01803, USA \\ M.Burlaka@softinway.com
}

SoftlnWay Turbomachinery Solutions Pvt Ltd.

Flat \# 301, 3rd Floor, Royal Manor, 70/10, Cunningham Road, Bangalore, KA, 560052 India Abdul.Nassar@SoftInWay.com

\author{
Petr Pagur \\ SoftInWay Inc. \\ 15 New England Executive Park \\ Burlington, MA01803, USA \\ Petr.Pagur@SoftlnWay.com
}

\author{
Dr. Leonid Moroz \\ SoftInWay Inc. \\ 15 New England Executive Park \\ Burlington, MA01803, USA \\ L.Moroz@SoftInWay.com
}

\author{
Dr. Yuri Govoruschenko \\ SoftInWay Inc. \\ 15 New England Executive Park \\ Burlington, MA01803, USA \\ i.govoruschenko@softinway.com
}

\begin{abstract}
The use of S-CO2 as working fluid in a power cycle has been growing in recent years due to associated benefits such as highly compact power plant and high cycle thermal efficiencies for application including waste heat, solar thermal and nuclear power plants. Many authors have presented studies on S-CO2 cycle and its modifications and there also exists many patents which claim different embodiments of the S-CO2 cycle for different heat sources. Each author of the S-CO2 cycle embodiment uses some specific tool to analyze the cycle performance with assumed values of component efficiencies. In the S-CO2 cycle the ratio of turbine work to compressor work is relatively small and its variation may cause a significant influence on cycle performance estimation accuracy. Exact prediction of the $\mathrm{S}-\mathrm{CO} 2$ cycle performance requires defining exact turbomachinery efficiency magnitudes. However, S-CO2 turbines and compressors are in development stage except for several low power scale prototypes and hence it is very difficult to make assumptions on efficiency and they need to be designed.
\end{abstract}

To enable design of cycle from concept to detailed design of the turbomachinery, the authors in this work have developed a flexible design system which is starting from heat balance calculation, continues with sizing of turbomachinery flow path, through $1 \mathrm{D} / 2 \mathrm{D} / 3 \mathrm{D}$ aero and structural multidisciplinary optimization. Such a design process is iterative because a refinement of the turbomachinery efficiencies lead to change in cycle boundary conditions for turbomachinery design and the design needs to be refined by recalculation of the cycle. In the present work, four different embodiments of S-CO2 thermodynamic cycles were analyzed using assumed component efficiencies and based on the actual design of the turbomachinery components the cycle was recalculated and accurate performance of the cycle was predicted. It is observed that the turbine efficiency has significant influence on the overall cycle performance compared to the compressor efficiency.

\section{INTRODUCTION}

In recent times, S-CO2 turbines has become a topic of hot research with many organizations and researchers working to meet the requirement for not only as a bottoming cycle but also as a topping cycle with the objective of replacing conventional steam and gas turbine power plants. Based on the heat source different types of cycles have been conceptualized as topping or bottoming cycles. Majority of the publications on $\mathrm{S}-\mathrm{CO} 2$ is focused on the thermodynamic cycle analysis and optimization with various embodiments proposed by different authors, each of them using a specific tool to analyze the cycle performance with assumed values of component efficiencies [1-3].

The ratio of turbine work to pump work in the Rankine cycle is quite large and only a turbine performance can cause a significant effect on cycle efficiency whereas in an S-CO2 cycle, ratio of turbine work to compressor work is relatively small and it causes a significant influence on cycle efficiency (i.e. it is necessary to correctly calculate not only a turbine's 
efficiency but also compressor's efficiency) [4]. The authors here have made an attempt to perform cycle analysis for $\mathrm{S}-\mathrm{CO} 2$ with assumed efficiencies and then based on the results of the cycle analysis the turbines and compressors for $\mathrm{S}-\mathrm{CO} 2$ are independently designed to obtain the actual efficiencies and then the cycle performance is recalculated with actual efficiencies all through a fully integrated interface.

Usually the supercritical $\mathrm{CO} 2$ power cycle is based on the closed recuperated Brayton cycle and its modifications, which include the pre-compression cycle, recompression cycle, and partial cooling cycle [5]. It is very important to study wide variety of the S-CO2 cycle embodiments to find the optimum one for each specific heat source and this requires use of flexible heat balance calculation tool and here the developed tool AxCYCLE is used to study three different embodiments based on [6] [7] [8]. The impact of efficiencies of compressor and turbine is also studied to determine the significance of component efficiencies on the overall cycle performance. Based on these results compressors and turbines were designed in the integrated approach and components matched for establishing operating line and then the cycle performance recalculated based on the efficiencies of the designed compressors and turbine.

\section{DESIGN SYSTEM}

Figure 1 depicts the flow process diagram of the integrated design system. The thermodynamic cycle is built by creating a schema by interactively dragging and dropping the components from the component palette of the developed system. Once the components are built, boundary conditions for each component are set along with assumed efficiencies of the component. The calculations are performed and results reviewed before performing off-design map calculations. As a result of the thermodynamic cycle analysis, the boundary conditions for designing the compressor and turbine are obtained. The next step is to perform preliminary design by specifying the geometrical constraints for sizing the flow path. Once preliminary flow path is obtained detailed 1D/2D calculations are performed before proceeding to profiling and blade design. As a final step off-design performance is estimated and maps generated individually for compressor and turbine and finally matched. At any stage during this process if the solution is not satisfactory, the design can be taken to the previous steps without leaving the interface. The thermodynamic cycle is recalculated based on the actual efficiencies that are obtained from the detailed design of the component and if significant change in performance is observed, the component can be redesigned for the new cycle conditions. As a result of this process, the total system for the S-CO2 can be designed all in a unified interface with minimal effort.

The thermodynamic cycle analysis system solves a system of algebraic equation to satisfy energy and mass balance as a network flow. For steady state models and for nodes the continuity equation is simplified to

$$
\sum_{\text {branches }} \dot{m}=0
$$

The energy balance for the component is applied in a general way as

$$
\begin{gathered}
\sum_{\begin{array}{c}
\text { mass } \\
\text { branches }
\end{array}} \dot{m} h+\sum_{\begin{array}{c}
\text { heat flow } \\
\text { branches }
\end{array}} \dot{Q}+\sum_{\begin{array}{c}
\text { Power } \\
\text { branches }
\end{array}} \dot{P}+\sum_{\begin{array}{c}
\text { Mechanical } \\
\text { branches }
\end{array}} \dot{W} \\
=\sum_{\text {fluids }} \frac{U}{d \tau}+\frac{U_{s}}{d \tau}
\end{gathered}
$$

where $\mathrm{h}$ - enthalpy of fluid, $\mathrm{Q}$ - heat flow, $\mathrm{P}$ - electrical power, $\mathrm{W}$ - mechanical power, $\mathrm{U}-$ the internal energy of fluid mass with specific internal energy and $U_{s}$ - internal energy of the material of the device [9].

The pressure drop is a function of several variables, $\Delta \mathrm{P}=$ $\mathrm{f}(\mathrm{m}, \Delta \mathrm{H}, \Delta \rho, \mathrm{P}, \mathrm{W})$. The solver provides the balance equation for the system during system identification. This feature not only eases the user's work and ensures that the system fulfills the balance equations; it also has made it possible to avoid singularities caused by cyclic steady-state processes, which are very common in energy system models. Backward Differentiation Formula (BDF) method for solving linear implicit and semi-explicit equations and Modified Newton Method for solving non-linear implicit equations are implemented in the solver of the thermodynamic cycle analysis tool AxCYCLE [9]. For setting up the equations for system identification the input data is categorized into parameter (which is a variable that depends on the component), fixed (a value that should be achieved during the process) and initial (approximate values that are initial guesses for the solver to begin calculation). Once the calculation is performed the solver provides heat and mass balance between the components along with the cycle performance in terms of efficiency and power output.

\section{THERMODYNAMIC CYCLE ANALYSIS}

Three different S-CO2 cycles embodiments are analyzed in this work and will be presented here. The first embodiment is a recompression cycle based on a patent of Letwinet al. [6] which is designed for a solar thermal S-CO2 based power plant cycle shown in Fig. 2. The second embodiment is based on the patent of Held [7] Hot Day Cycle which is based on a simple closed recuperated $\mathrm{S}-\mathrm{CO} 2$ cycle for application in waste heat recovery and the third embodiment is partial cooling power cycle presented by Kulhanek \& Dostal [8].

The recompression cycle of S-CO2 turbine system includes two compressors (main compressor (1-2) \& recompression compressor (11-12), turbine (6-7), heater (5-6) High Temperature (HT) recuperator (7-8) Low Temperature (LT) recuperator (8-9) and cooler (10-1), and flow splitters (12-3 \& 9-10). An alternator is connected to the turbine shaft for power generation. The heat from the molten salt of the solar cycle is transferred to the S-CO2 by the heater and the working fluid is 
cooled before entering into the main compressor by cooler. Internal efficiency for the compressor is considered as $89 \%$ and for the turbine internal efficiency is assumed to be $85 \%$. Additional input provided are the temperature for the fluid at the heat source $(550 \mathrm{C})$ and required temperature at the outlet of the LT - recuperator $(70 \mathrm{C})$.

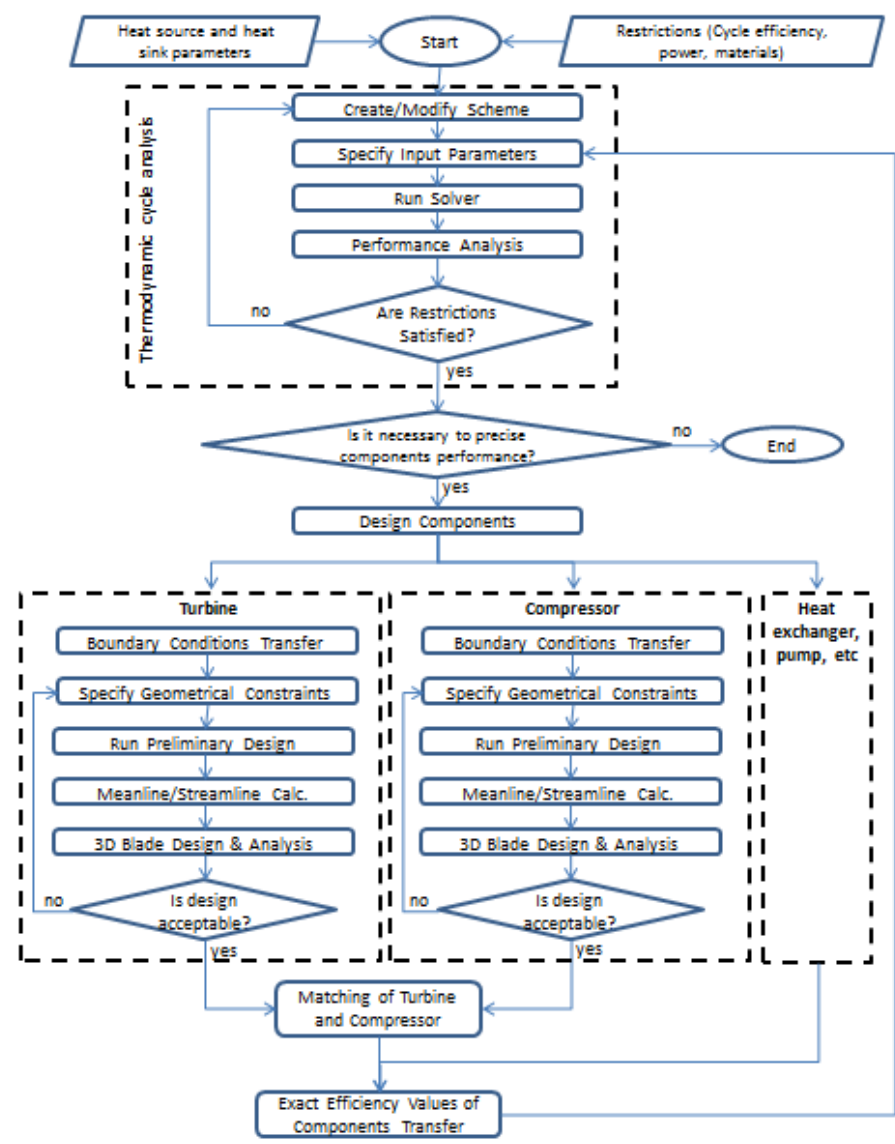

Figure 1. Flow chart of the integrated design system.

\section{Recompression Cycle}

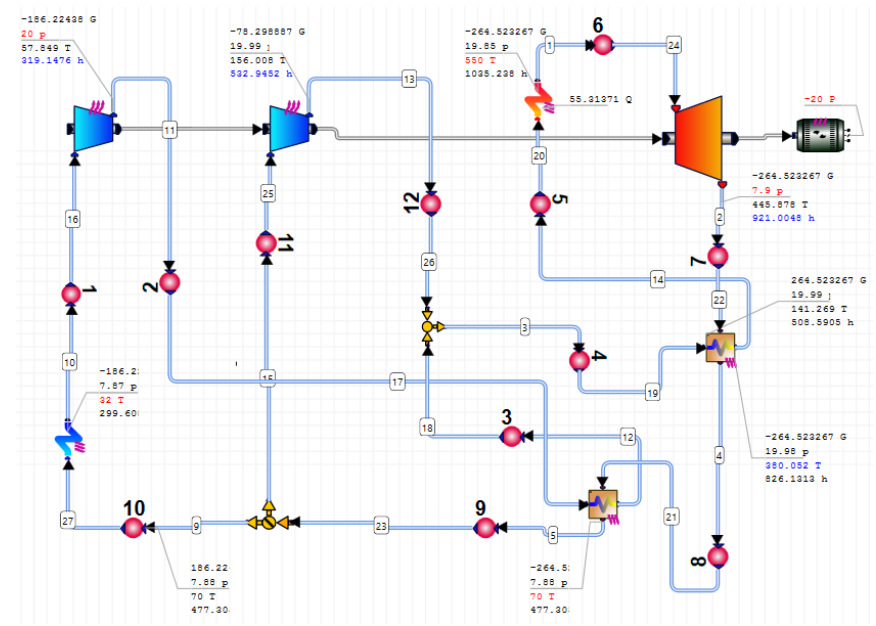

Figure 2. Recompression S-CO2 cycle with results

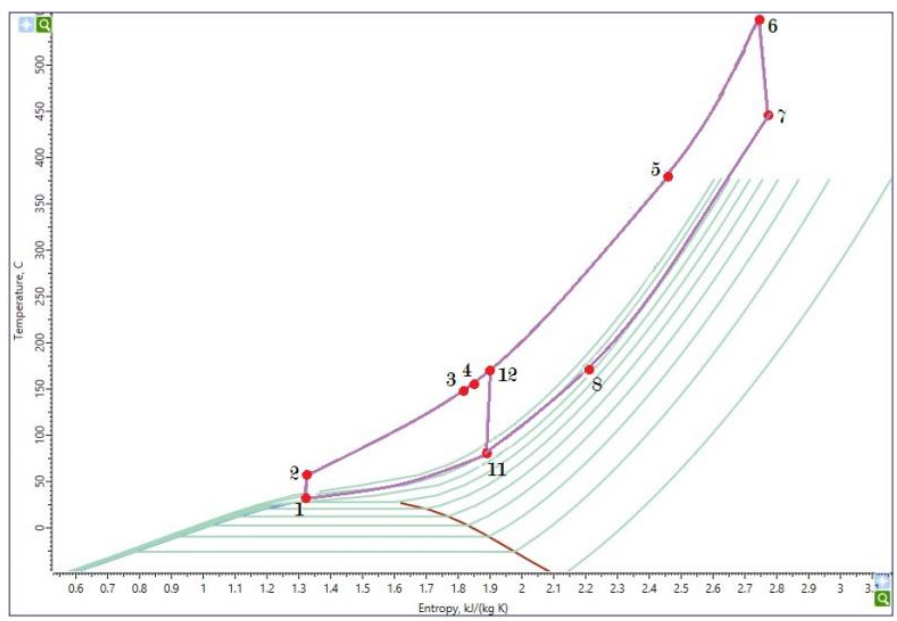

Figure 3. T-S diagram for Recompression cycle

The heat and mass balance diagram is presented in Figure 2. The data presented is in the following order, mass, pressure, temperature and enthalpy. The component thermodynamic data becomes the basis for design of the component. The $\mathrm{T}-\mathrm{S}$ diagram for the recompression cycle is shown in Figure 3. The compression process in main compressor is represented by the line (1-2), the compression process in recompression compressor represented by line (11-12) and expansion process in the turbine is represented by the line (6-7). The process from (2-6) represents the heat addition in the system and process from (7-11) and (11-1) represents heat rejection in the process. The efficiency of a thermodynamic process is a ratio of the useful output to the input, both measured in terms of energy. This particular recompression cycle produces $20 \mathrm{MW}$ of electrical power with an overall cycle efficiency of $36.1 \%$.

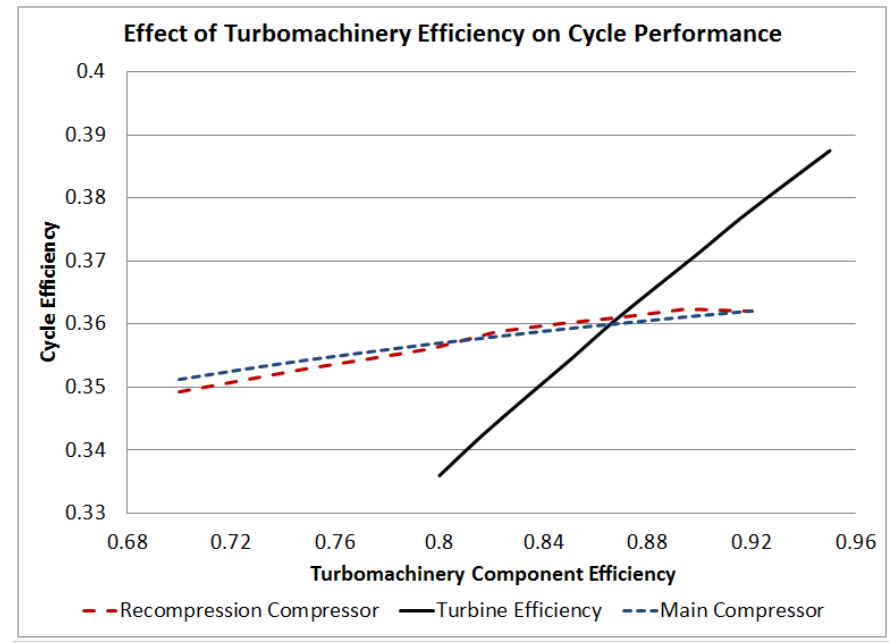

Figure 4. Effect of compressor (main and recompression) and turbine efficiency on cycle efficiency

To study the effect of compressor and turbine efficiencies on the performance of the cycle, the efficiencies of turbine, main compressor and recompression compressor was varied in a range independently and the results are plotted in Figure 4. 
The turbine efficiency changes has a significant change on the cycle performance compared to compressor efficiency which provides a basis that slight variation in turbine efficiency can significantly change the overall cycle efficiency.

\section{Simple CO2 Brayton cycle with recuperator}

The second cycle based on Held [7] is designed considering the waste heat from gas turbine as the heat source for the S-CO2 cycle based on the prior art depicting a hot day cycle as given in the patent. This cycle is designed as a Brayton cycle with recuperator and the cycle is shown in Figure 5. As an input, the efficiency of the turbine is considered as $90 \%$. In this cycle a pump (1-2) is used instead of a compressor because the magnitudes of pressure and temperature at the lower side are below that of the critical values. The internal efficiency of the pump is taken as $92 \%$. Appropriate pressure loss for the heater (3-4), recuperator (5-6) and cooler (6-1) are considered during the analysis. Due to lack of information available on heat exchanger effectiveness and pressure loss fixed values were considered and these have not been recalculated in this work.

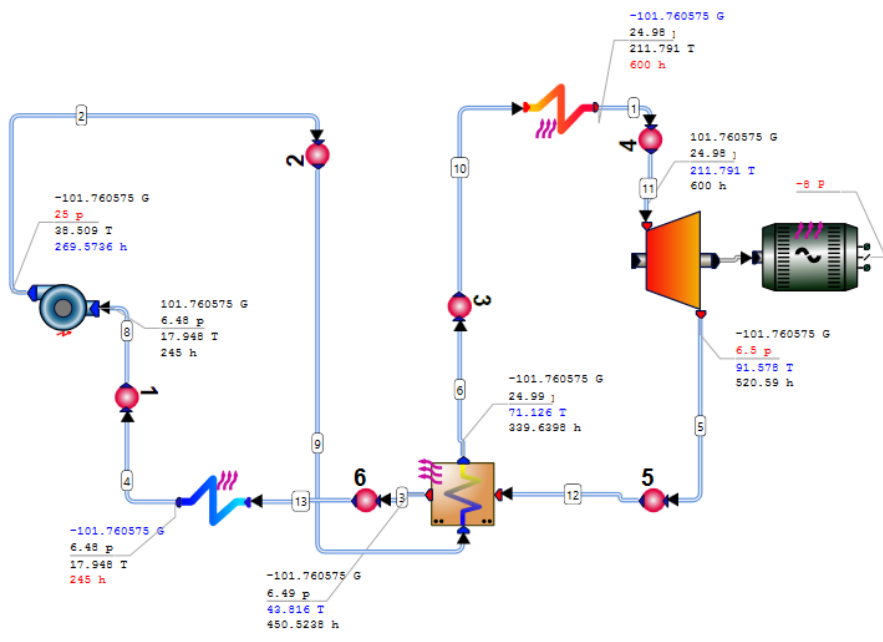

Figure 5. Brayton Cycle with Recuperator\& Pump for CO2

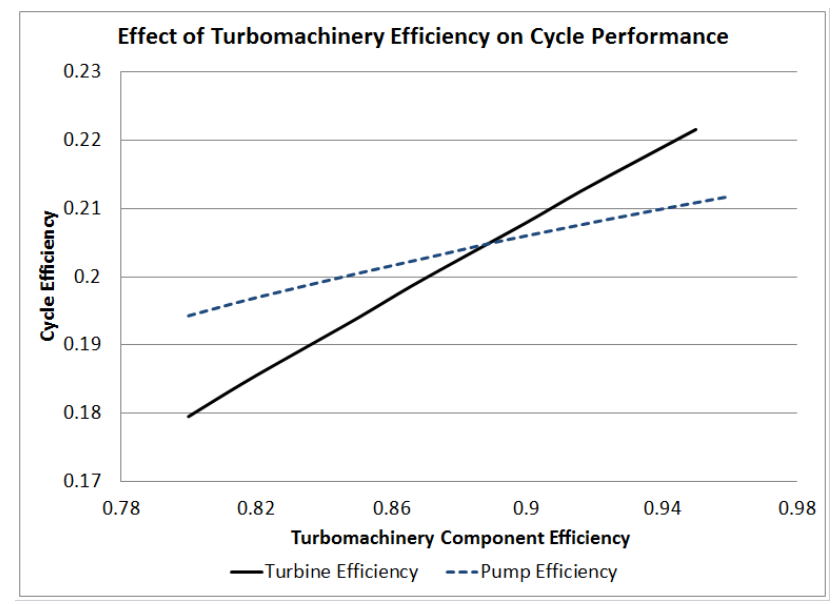

Figure 6. Effect of pump and turbine efficiency on cycle efficiency
The CO2 after the cooler is in a liquid state and the cooler is placed in the two phase region. The fluid from the pump is pumped to the recuperator before passing through the heater and then enters into the turbine. The fluid after expanding from the turbine passes through the recuperator exchanging the heat from turbine exhaust to the fluid before entering the heater and is then cooled at the cooler (condenser) before entering the pump and the cycle continues. The efficiency of a thermodynamic process is a ratio of the useful output to the input, both measured in terms of energy. The overall cycle efficiency was found to be $20.8 \%$ with a net power produced is 6.78 MW. From Figure 6, it is clear the turbine has a larger influence on the cycle performance compared to the pump.

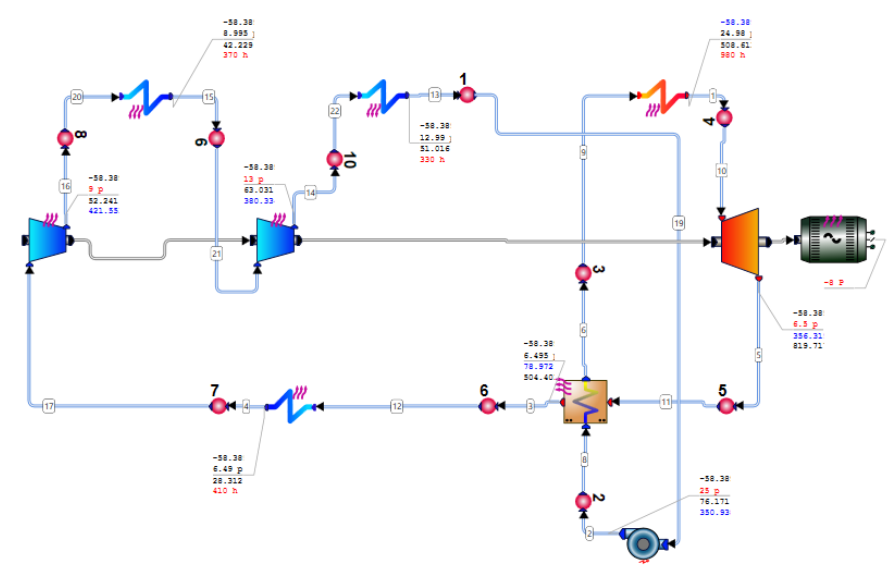

Figure 7. Brayton Cycle for $\mathrm{CO} 2$ with compression and cooling

Due to the poor efficiency of this cycle, another embodiment of Held [7] considering an environment that has high temperatures is shown in Figure 7. The objective is to improve efficiency of the cycle, retain the same power output, but reduce the heat transferred to the cycle from source.

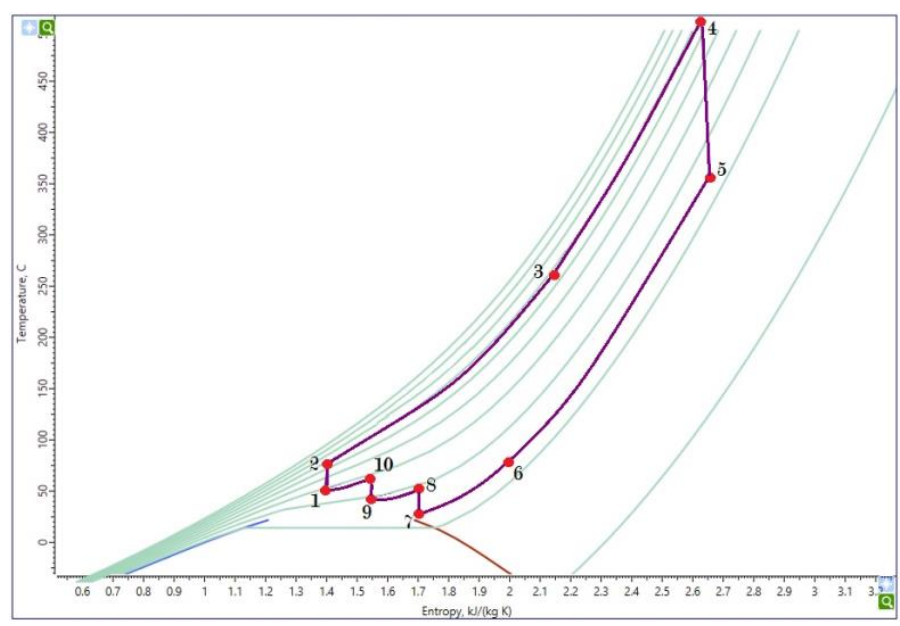

Figure 8. T-S diagram for S-CO2 Brayton Cycle with recuperator modified with compression and cooling

This cycle uses sequential cooling and compression of CO2. The pump (1-2) pumps the fluid through a recuperator (2- 
3) and passes through the heat source (3-4) and then expands in the turbine (4-5), loses some heat at the recuperator and is then further cooled by the cooler (6-7) before entering into the first compressor (7-8). The compressed fluid is then subsequently cooled in cooler (8-9) and again compressed in next compressor (9-10) and further cooled in the next cooler (10 -1) before entering the pump and the process continues. Both the compressors were considered to be $89 \%$ efficient. In the second embodiment, the power output was maintained at $6.78 \mathrm{MW}$ and the cycle efficiency increased to $37.27 \%$ which shows that with sequential compression and cooling has significant decrease in source heat or fuel consumption.

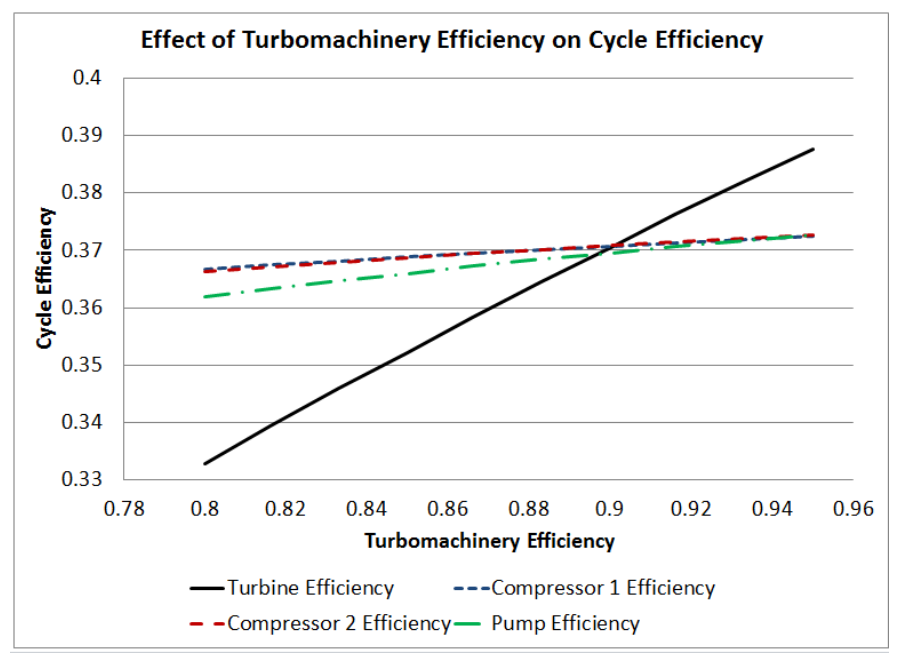

Figure 9. Effect of efficiency of compressor, turbine and pump on overall cycle

Figure 8 shows the T-S diagram for S-CO2 based Brayton Cycle with recuperator modified to include compression and cooling. The process (1-2) represents the pump work, process (2-3) is heat addition in the recuperator, (3-4) is heat addition in the heater (heat source from gas turbine) and (4-5) represents expansion in the turbine. The heat rejection from (5-6) happens in the recuperator and (6-7) in cooler by condensation. Subsequently the compression takes place from (7-8) \& (9-10) and cooling in (8-9) \& (10-1). The effect of variation in efficiency of the compressor, pump and turbine is plotted in Figure 9. Turbine efficiency plays a significant role in overall performance of the cycle compared to compressor and pump.

\section{Partial Cooling Power Cycle}

This embodiment is based on the work of Kulhanek \& Dostal [8] for which the heat source is considered as nuclear reactor and is shown in Figure 10. In this cycle $\mathrm{CO} 2$ is expanded in the turbine up to a pressure below critical and then an additional compressor is used to raise the pressure above critical value and after that the flow is separated into two parts with one part compressed up to top cycle pressure and the other part is cooled and then compressed. The fluid is first compressed in the compressor (1-2) and then is heated through the recuperator (2-3) and enters the mixer located between (3-4) where a part of the fluid that is compressed in compressor (1213) to the top cycle pressure mixes and flows to the next recuperator (4-5) before entering the heat source (5-6) and then expands in the turbine (6-7). The fluid that is expanded in the turbine flows through the two recuperators and then is cooled further in the cooler (9-10) and compressed in compressor (1011) after which the fluid is split into two parts with one part entering the cooler (14-1) and the other part to the compressor (12-13) as discussed above.

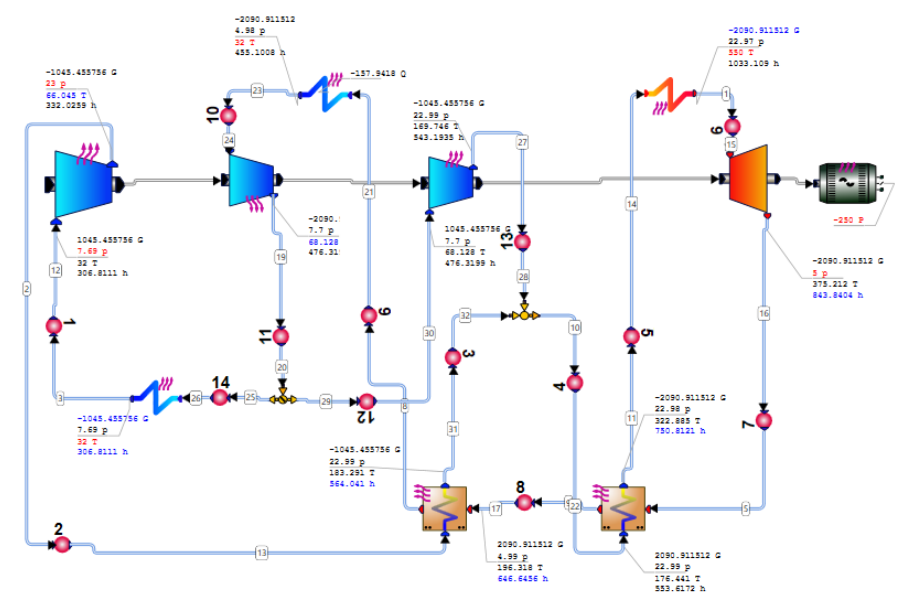

Figure 10. S-CO2 for nuclear reactor

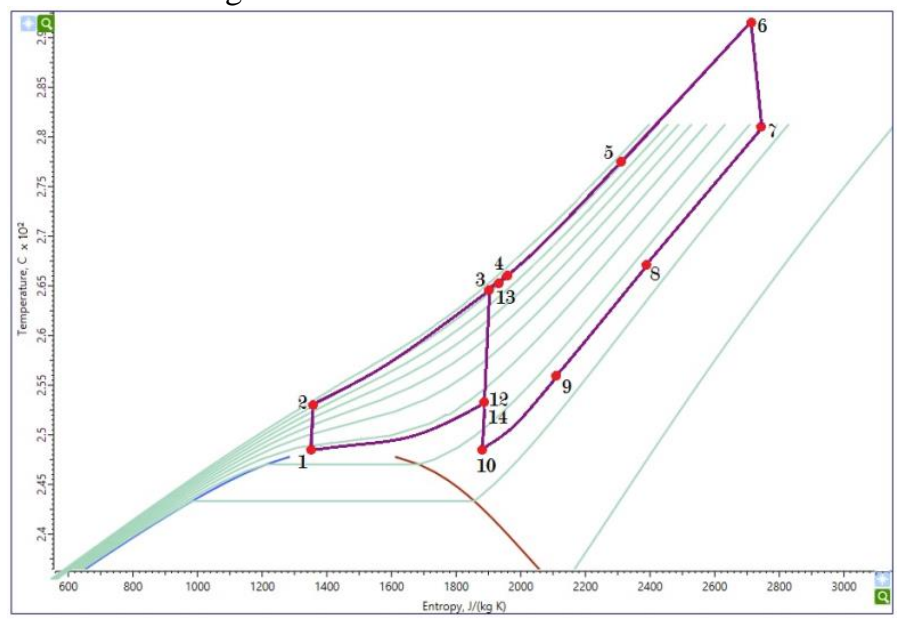

Figure 11. T-S diagram of S-CO2 for nuclear reactor

The T-S diagram shown in Figure 11 shows the process of compression, expansion, heating and cooling. The process (1-2) represent the compression process, (2-3) heating in recuperator and mixing of part of compressed fluid extracted before cooling in the cooler at $(3,4,13)$ and further heating in recuperator (45 ) and heating at heat source (5-6) and then expansion in turbine (6-7). The exhaust from turbine passes through the recuperator (7-8 \& 8-9) and then is cooled in cooler (9-10) before compressing in compressor (10-11) after which a part of the fluid is compressed to the system top pressure and mixes at $(3,4,13)$ and the other part is cooled further in cooler (10-1) before compressing and the cycle continues. 


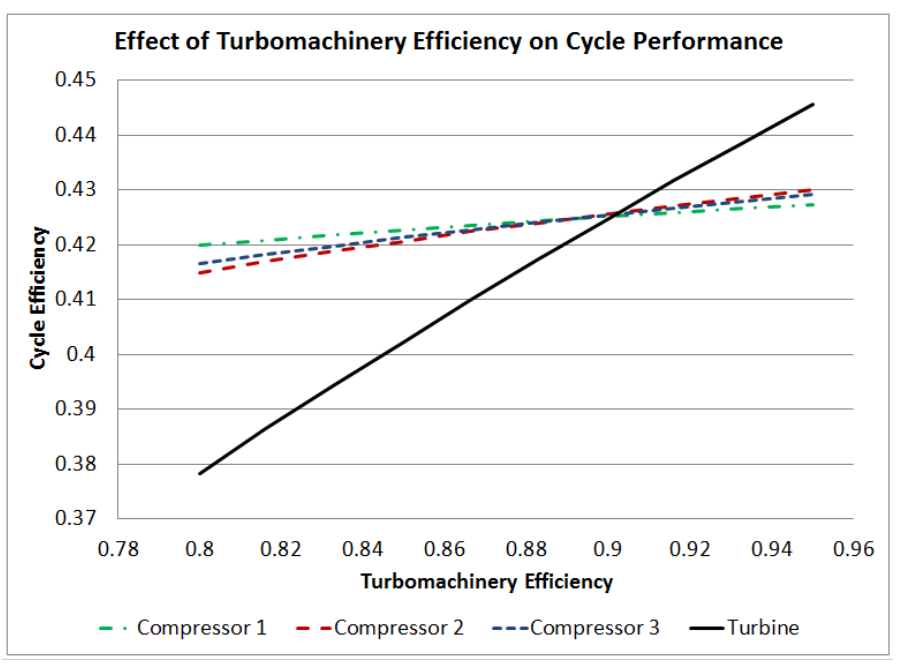

Figure 12. Effect of efficiency of compressor and turbine on overall partial cooling power cycle

Similar to the other cycles, the variation of turbine efficiency is significant on the overall performance of the cycle as observed from Figure 12. Though not very significant, if each of the compressor efficiency is off by more than $2-3 \%$, they can significantly affect the overall performance of the cycle. Hence it is very critical to accurately provide actual efficiencies to get more realistic calculation of the cycle. The overall efficiency of the cycle was $42.35 \%$.

The cycle analysis was performed without considering the type of turbomachinery, structural aspects and geometric dimensions. When a new cycle is being designed where we don't have the turbomachinery designed then it is preferable to design the turbomachinery after cycle analysis and recalculate the cycle performance with actual efficiencies that are obtained from the designed turbomachinery. Here the authors have used turbomachinery design and analysis suite AxSTREAM ${ }^{\mathrm{TM}}$ which has been used successfully for designing turbomachinery from conceptual flowpath to 3D blades [10] [11]

\section{DESIGN OF TURBINE FOR S-CO2}

From the cycle analysis the inlet and outlet boundary conditions such as inlet pressure and enthalpy, outlet pressure and mass flow rate are obtained based on which the turbine has to be designed. The turbine design process starts by sizing the flow path for a given boundary conditions and performing a meanline analysis as the first step. The design process implemented here use a fast meanline solver for preliminary sizing and design evaluation, and increase complication with axisymmetric and quasi-3D codes, while it's moving from general concepts to particular machine details on higher design process levels. The detailed theoretical background and structure of preliminary design and sizing part of the system is provided in [12].

To obtain an optimal flow path [13], the designer has to arrive at optimal isentropic velocity ratio (U/C0), flow path dimensions, number of stages, etc. Also decision has to be done on choosing the turbine design type as impulse/reaction or combination of both.

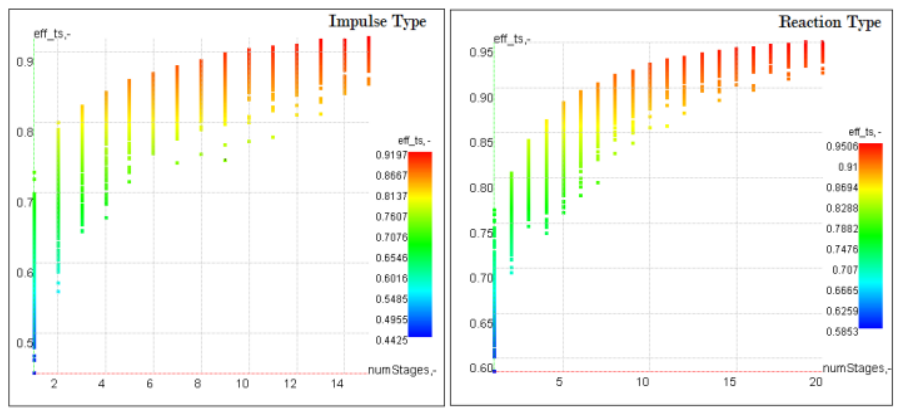

Figure 13. Effect of number of stages on total-to-static efficiency for impulse and reaction type turbine design

The boundary conditions from the cycle analysis was specified in the preliminary design module (refer Figure 1) and flow path geometry obtained. The rotational speed was taken as $3000 \mathrm{rpm}$ for the turbine and preliminary design performed to analyze the type of turbine to be designed and arrive at optimal number of stages. From Figure 13, it is observed that the efficiency is higher for reaction machine and as the number of stages increases the efficiency increases but at the cost of axial length, capital investment and start up time. Evaluating different parameters like axial length, incremental improvement in efficiency, blade mass, diameters, $\mathrm{D} / \mathrm{L}$ ratios and manufacturability versus number of stages, it was decided to further evaluate a 7 stage impulse turbine and 11 stage reaction turbine.
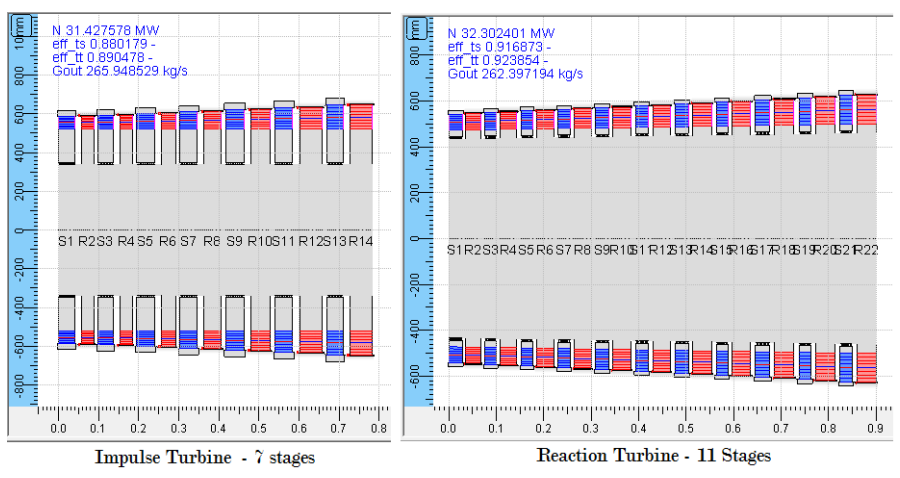

Figure 14. Flow path of impulse and reaction type turbine after adjusting flow path dimensions and performing streamline calculations

Modified Crag \& Cox loss model by the authors company implemented in the design tool was used for the detailed design of the flow path for both impulse turbine and reaction turbine. S1 Optimization [13] was performed to obtain the optimal chord length and relative pitch for all the stages by considering not only the aerodynamic performance but also including the structural constraints. The structural calculation during S1 optimization is based on 1D beam element theory due to which appropriate design safety factor needs to be considered [14]. 
Appropriate design safety factors were considered for the blade bending stresses, blade yield strength and creep strength based on authors experience in performing design for similar type of turbomachinery.

Table1: Comparison of impulse and reaction design turbine

\begin{tabular}{|l|c|c|c|}
\hline & $\begin{array}{l}\text { From Cycle } \\
\text { analysis }\end{array}$ & $\begin{array}{l}\text { Impulse } \\
\text { Design }\end{array}$ & $\begin{array}{l}\text { Reaction } \\
\text { Design }\end{array}$ \\
\hline Efficiency & 0.85 & 88.02 & 91.68 \\
\hline Power $(\mathrm{MW})$ & 30.17 & 31.43 & 32.30 \\
\hline Axial Length $(\mathrm{mm})$ & NA & 783 & 903 \\
\hline No of stages & NA & 7 & 11 \\
\hline Mass flow rate $(\mathrm{kg} / \mathrm{s})$ & NA & 265.94 & 262.39 \\
\hline
\end{tabular}

The flow path of the 7 stage impulse and 11 stage reaction turbine after streamline calculation and profiling is presented in Figure 14. Due to higher loading in impulse turbine the chord length were larger compared to reaction machine and due to this the overall length variation is only $120 \mathrm{~mm}$. Review of the axial load on both the turbines showed reaction turbine has 2.5 times more axial load compared to the impulse turbine and would require a balancing piston which can lead to increase in flow path length. Table 1 shows the comparison of impulse and reaction design turbine with reference value of performance from cycle analysis. The maximum bending stress acting on the rotating blades are presented in table 2 for both impulse and reaction turbine stages.

Table 2: Maximum blade bending stress in rotating blades for impulse and reaction turbine stages

\begin{tabular}{|c|c|c|}
\hline \multicolumn{3}{|c|}{ Maximum bending stress in rotor blades (MPa) } \\
\hline Stage \# & Impulse Turbine & Reaction Turbine \\
\hline 1 & 31.90 & 35.38 \\
\hline 2 & 32.71 & 32.91 \\
\hline 3 & 28.97 & 31.45 \\
\hline 4 & 21.63 & 33.62 \\
\hline 5 & 20.92 & 31.84 \\
\hline 6 & 17.53 & 34.05 \\
\hline 7 & 22.05 & 32.92 \\
\hline 8 & & 35.31 \\
\hline 9 & & 32.08 \\
\hline 10 & & 34.64 \\
\hline \multirow{2n}{*nn}{} & & 37.36 \\
\hline
\end{tabular}

The Mollier diagram for the impulse and reaction turbine is shown in Figure 15. Uniform heat drop between stages has been maintained for both impulse and reaction stages. The blue line indicates the heat drop in stator and the red line indicate heat drop in rotor. The isentropic heat drop between stages for impulse and reaction stages are shown in Figure 16. Impulse stages are normally loaded higher than reaction stages, the distribution of work coefficient for impulse and reaction stages are also plotted in Figure 16. The average work coefficient in impulse design is 2.1 whereas for reaction design it is 1.5 .The distribution of velocity ratio and flow coefficient for impulse and reaction stages is plotted in Figure 17.
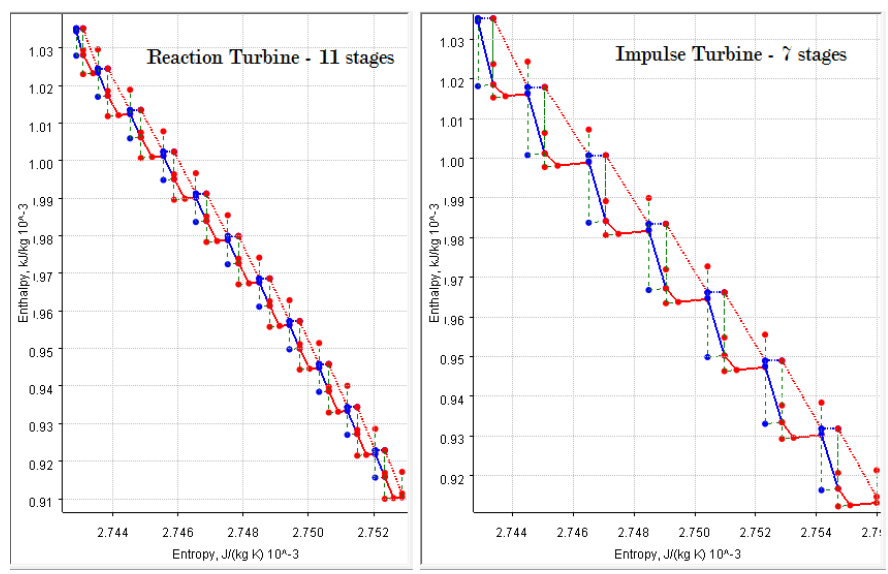

Figure 15. Heat drop distribution in stages for impulse and reaction type design

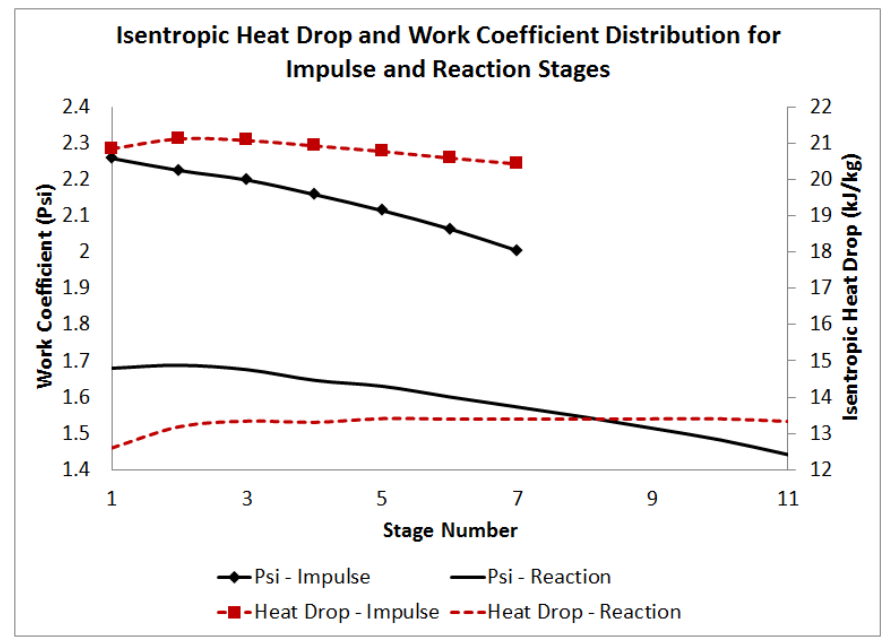

Figure 16. Isentropic Heat Distribution and Work Coefficient distribution

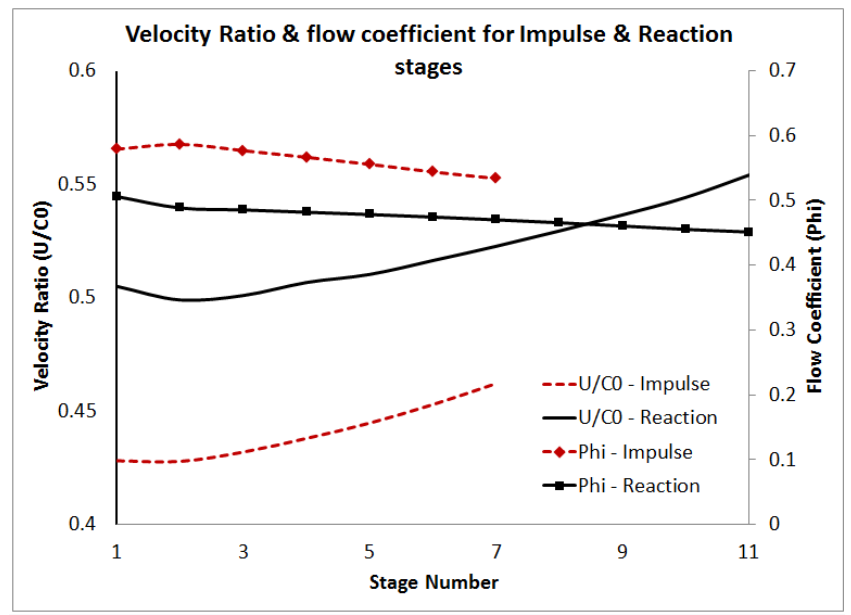

Figure 17. Velocity Ratio and Flow Coefficient for impulse and reaction stages

The 3D geometry of the flow path is shown in Figure 18. The integrated design system can be used not only for 
generating the flow path and blade profiles, but also for designing the attachment which is shown in Figure 18.
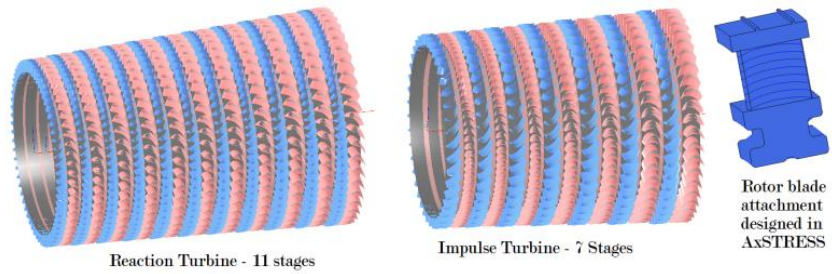

Figure 18. The 3D geometry of the flow path \& single rotor blade with attachment designed in AxSTRESS

\section{DESIGN OF COMPRESSOR FOR S-CO2}

Based on the specification obtained from thermodynamic cycle analysis, two compressors, one as main compressor and other as recompression compressor needs to be designed. Though both compressors are for same pressure ratio, they have different mass flow requirements and thus necessitate two different designs. The authors here performed conceptual study for both main compressor and recompression compressor considering axial and centrifugal type and the results are presented here.

\section{Axial Compressor Design}

The preliminary design was performed to study axial compressor flow path for the main compressor and arrive at optimal number of stages, speed and flow path dimensions. Figure 19 (a) shows efficiency as a function of number of stages and speed and Figure 19 (b) shows min blade height as a function of number of stages and speed. From the plots it is observed that $\mathrm{S}-\mathrm{CO} 2$ being denser fluid, the blade heights are quite small which makes manufacturing process complicated. Since the mass flow in main compressor is 2.37 times higher than the recompression compressor, the blade height for recompression compressor would be much smaller resulting into unrealistic flow path dimensions. The resultof recompression compressor is not presented here.

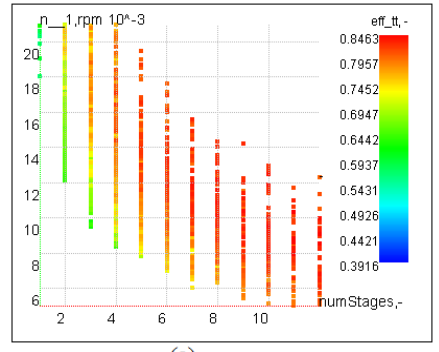

(a)

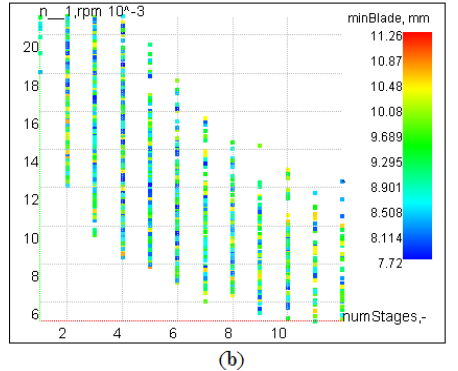

Figure 19. Different designs plotted in terms of efficiency and minimum blade height as a function of number of stages and rotational speed

The flow path of the axial configuration of the main compressor consisting of 12 stages and operating at a speed of $12333 \mathrm{rpm}$ is shown in Figure 20. By reducing number of stages and increasing last stage blade height to a minimum of $10 \mathrm{~mm}$ results in significant decrease in efficiency. This makes axial flow path configuration not a right choice for this requirement and centrifugal compressor design were evaluated.

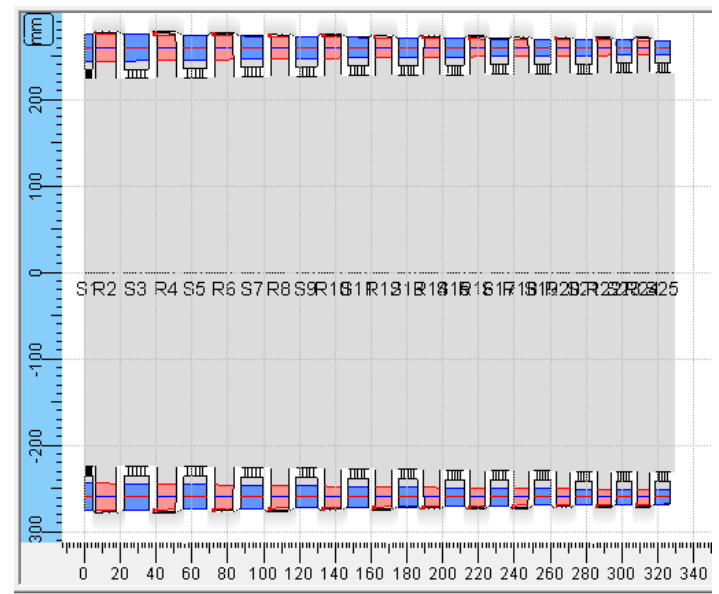

Figure 20. Flow path of axial configuration of the main compressor at best efficiency consisting of 12 stages and operating at $12333 \mathrm{rpm}$

\section{Centrifugal Compressor Design}

The next choice was to perform preliminary design on centrifugal compressor flow path type for the main compressor and arrive at optimal speed and flow path dimensions. Since the pressure ratio required is 2.53, a single stage centrifugal compressor would suffice this requirement. As a first step the main compressor and recompression compressor was designed for a range of speeds starting from $3000 \mathrm{rpm}$ to $21000 \mathrm{rpm}$, the results are plotted in Figure 21. Since the turbine speed is 3000 rpm, and no feasible solutions were obtained for compressor speed of $3000 \mathrm{rpm}$, it was decided to review both the compressor performance for a particular speed and use same speeds for both the compressor which will enable mounting them on single shaft connected to a gearbox and coupled to the turbine. Both the compressors had maximal efficiency in the range of $20,400-20,700 \mathrm{rpm}$, additional calculations were performed and design speed selected as 20,500 rpm.

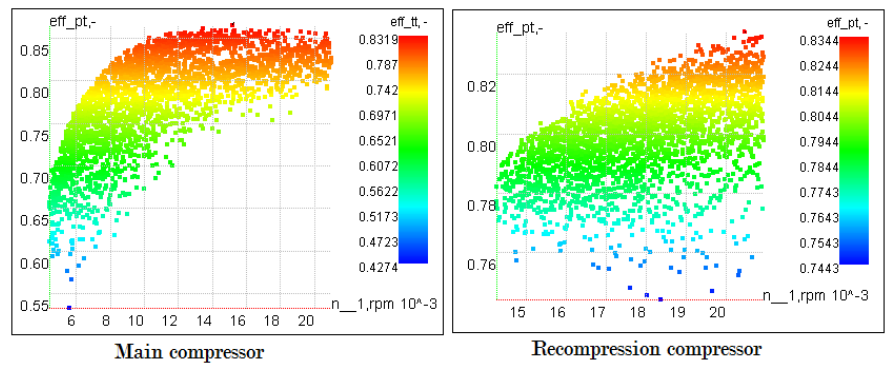

Figure 21. Design designs plotted to compare efficiency versus speed for main compressor and recompression compressor

The flow path of the main compressor and recompression compressor is shown in Figure 22. Due to higher specific speed of the main compressor, the axial length of the flow path is 
shorter compared to the recompression compressor. From streamline calculations after profiling the main compressor had internal total-to-total efficiency of $81.67 \%$ and recompression compressor with $82.30 \%$ which is significantly lower than the initially assumed value of $89 \%$ in the cycle calculations. The velocity triangle for both compressors is shown in Figure 23.
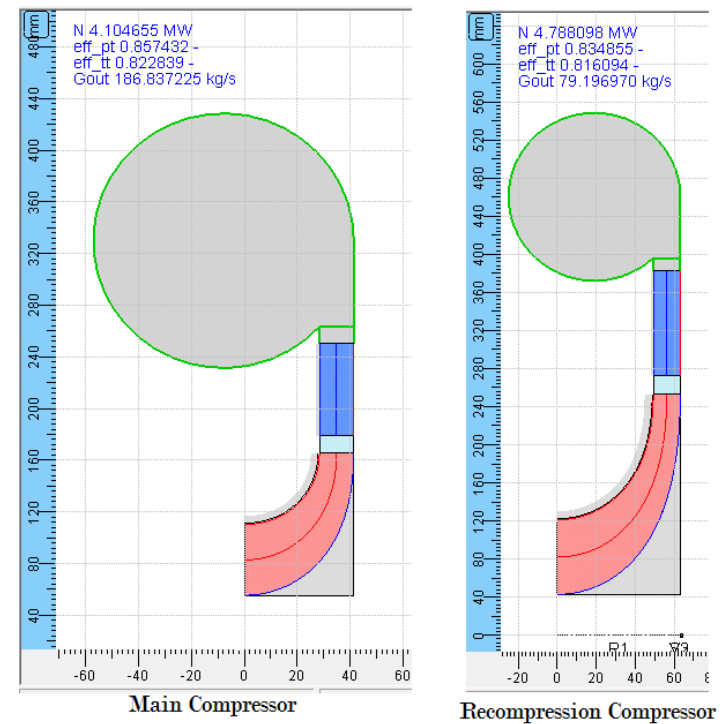

Figure 22. Flow path configuration of (a) main compressor and (b) recompression compressor
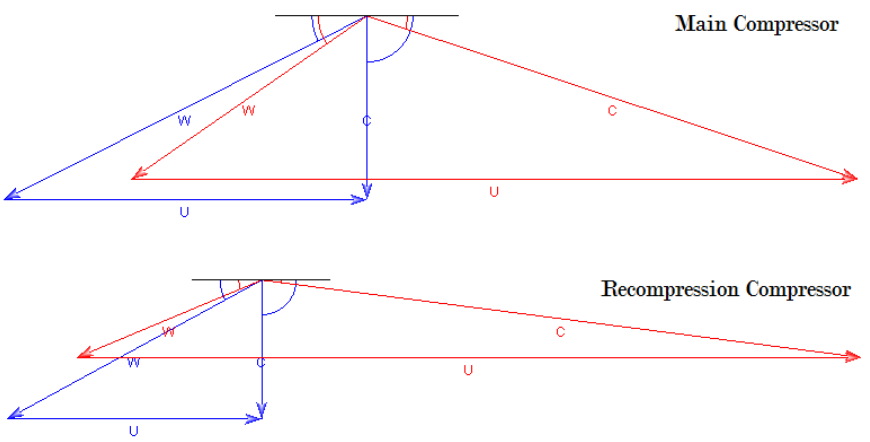

Figure 23. Velocity triangle at mean section of (a) main compressor and (b) recompression compressor

\section{RECALCULATION OF THERMODYNAMIC CYCLE AFTER COMPONENT DESIGN}

After performing detailed design of the turbine and compressor the actual efficiencies obtained are significantly different from the assumed efficiencies applied during the thermodynamic cycle analysis. The turbine efficiency is higher both for impulse design and reaction design compared to the assumed value of $85 \%$ and compressor efficiency is lower for both the main compressor and recompression compressor. To obtain the actual thermodynamic performance of the cycle, the cycle was recalculated with the actual designed efficiencies and resulted in an increase of $0.38 \%$ from the initial value for the impulse type turbine and $1.6 \%$ increase from initial value when reaction type turbine was considered.

\section{CONCLUSIONS}

Supercritical $\mathrm{CO} 2$ power cycle is an emerging and promising technology for heat energy conversion for electricity or mechanical power.

- To perform the analysis of different embodiments of the supercritical $\mathrm{CO} 2$ cycle it is necessary to have a special flexible heat balance calculation tool.

- The simulation in AxCYCLE of different S-CO2 cycle embodiments, such as the recompression cycle for Concentrated Solar Power, nuclear reactor, simple recuperated Brayton cycle and cycle with sequential cooling and compression for waste heat showed that this tool allows quick and precise calculations of the cycle performance in a wide variety of schemes.

- Design and structural analysis of turbomachinery for recompression cycle for CSP showed that the assigned values of turbomachinery efficiencies at step of cycle analysis differ from the calculated ones in AxSTREAM. Thus engineers have the capability to obtain more accurate cycle performance prediction by correcting turbomachinery internal efficiencies.

- AxSTREAM allows selection of appropriate turbine type, main compressor type as well the recompression compressor type in a quick and efficient manner providing multi-parameter analysis during preliminary design.

- Reaction turbine provided better efficiency compared to impulse turbine for $\mathrm{S}-\mathrm{CO} 2$ with moderate increase in axial length.

- The turbomachinery analysis showed that it is necessary to use a reduction gear for both compressors in order to achieve high efficiency and as a result to achieve high overall cycle performance.

- Improving the performance of a cycle requires detailed study on the components to be used in the cycle.

- To improve performance of a given cycle, it is necessary to choose appropriate compression and cooling requirements for a given heat source and given power output.

\section{ACKNOWLEDGMENTS}

We wish to thank many people from SoftInWay Inc. team, who generously contributed with their time and effort in the preparation of this work. The strength and usability of the material, presented here are only as good as the inputs, and their insightful contributions are greatly appreciated. 


\section{REFERENCES}

[1] Steven A. Wright, Thomas M. Conboy, and Gary E. Rochau, "Overview of Supercritical CO2 power cycle development at Sandia National Laboratories", 2011 University Turbine Systems Research Workshop, October 25-27, 2011 Columbus, Ohio

[2] Moisseytsev, A.; Sienicki, J. J. "Performance improvement options for the supercritical carbon dioxide brayton cycle.”, Nuclear Engineering Division, Argonne National Laboratory, June 6, 2007

[3] Yang Chen, "Thermodynamic Cycles Using Carbon Dioxide as Working Fluid," Doctoral Thesis, School of Industrial Engineering and Management, Department of Energy Technology, October 2011

[4] Oleg Guriev, Yuriy Govoruschenko, Leonid Moroz, "Supercritical CO2 Brayton Cycle. S-CO2 represents an advance in power conversion technology", Turbomachinery International. The Global Journal of Energy Equipment, January/February 2013

[5] Dostal, V., "A Supercritical Carbon Dioxide Cycle for Next Generation Nuclear Reactors," Dissertation, Massachusetts Institute of Technology, Department of Nuclear Engineering, January 2004.

[6] Litwin Robert Z, Zillmer Andrew J., Hoffman Nathan J., Von Arx Alan V., "Supercritical C02 turbine for use in solar power plants” EP 1930587 A2.

[7] Timothy James Held, Hot day cycle - US 2013/0113221 A1.

[8] Kulhanek M., Dostal V., "Thermodynamic Analysis and Comparison of Supercritical Carbon Dioxide Cycles". Supercritical CO2 Power Cycle Symposium, May 24-25, 2011, Boulder, Colorado

[9] AxCYCLE user documentation, 2014, SoftInWay Inc.

[10] Leonid Moroz, Yuriy Govoruschenko, Petr Pagur, "A uniform approach to conceptual design of axial turbine / compressor flow path", The Future of Gas Turbine Technology. $3^{\text {rd }}$ International Conference, October 2006, Brussels, Belgium

[11] Leonid Moroz, Yuriy Govoruschenko, Petr Pagur, "Axial turbine stages design:1D/2D/3D simulation, experiment, optimization", Proceedings of ASME Turbo Expo 2005, Reno-Tahoe, Nevada, USA, GT2005-68614

[12] Leonid Moroz, Yuriy Govoruschenko, Petr Pagur, Kirill Grebennik, Wolfgang Kutrieb and Mike Kutrieb, "Integrated Environment for Gas Turbine Preliminary Design", International Gas Turbine Congress 2011, Osaka, Japan, IGTC2011-0007

[13] Boyko A. V., Govorushchenko Y. N., 1989, "Theoretical Basis of Axial-Flow Turbines Optimal Design", Kharkov, VishaShkola (in russian)

[14] Oleg Guriev, Leonid Moroz, Boris Frolov, Maksym Burlaka, "Turbomachinery Flow path design and performance analysis for supercritical CO2" Proceedings of ASME TurboExpo 2014, Dusseldorf, Germany GT2014-25385 